Pacific Journal of Mathematic 


\title{
ASCOLI'S THEOREM FOR SPACES OF MULTIFUNCTIONS
}

\author{
Y.-F. Lin and David A. Rose
}

The purpose of this paper is to prove that if $X$ and $Y$ are two arbitrary topological spaces and if $M(X, Y ; c)$ denotes the space of all multi-valued functions on $X$ to $Y$ with the compact-open topology, then a closed set $\mathscr{F} \subset M(X, Y ; c)$ is compact if at each point $x \in X, \mathscr{F}(x)=\cup\{F(x) \mid F \in \mathscr{F}\}$ has a compact closure in $Y$, and $\mathscr{F}$ is evenly continuous.

The classical theorem of Ascoli [1] asserts that a uniformly bounded, equicontinuous family of functions has a compact closure in the space of continuous functions with the topology of uniform convergence. This theorem has been the center of many papers, notably the works of Gale [2], Myers [5], Weston [6], and Morse-Kelley [3, pp. 233-237]. The purpose of this paper is to establish a similar version of the Ascoli theorem in the space of multi-valued functions.

Let $X$ and $Y$ be two nonvoid topological spaces. Then $F \subset X \times Y$ is said to be a multifunction on $X$ to $Y$, denoted by $F: X \rightarrow Y$, if and only if for each $x \in X$,

1.1 .

$$
\pi_{2}((\{x\} \times Y) \cap F) \neq \square,
$$

where $\pi_{2}$ is the second projection of $X \times Y$ onto $Y$, and $\square$ denotes the empty set. We shall write $F(x)$ for the set defined in 1.1. Thus, loosely speaking, a multifunction is a point-to-set correspondence. If $A$ and $B$ are subsets of $X$ and $Y$ respectively, then we denote $F(A)=\cup\{F(x) \mid x \in A\}$ and $F^{-1}(B)=\{x \in X \mid F(x) \cap B \neq \square\}$.

DeFinition 1.2. A multifunction $F: X \rightarrow Y$ is continuous if and only if for each open set $V$ in $Y$, the set $F^{-1}(V)$ is open and the set $F^{-1}(Y-V)$ is closed in $X$.

Let $Y^{X}$ be the set of all (single-valued) functions on $X$ to $Y$, and let $M(X, Y)$ be the set of all multifunctions on $X$ to $Y$. Note that $Y^{X}$ is a subset of $M(X, Y)$. For our later convenience in expressions, let us agree that for any $A \subset X$ and $B \subset Y$ :

$$
\begin{aligned}
& (A, B)=\{F \in M(X, Y) \mid F(A) \subset B\}, \\
& ) A, B\left(=\left\{F \in M(X, Y) \mid A \subset F^{-1}(B)\right\} .\right.
\end{aligned}
$$

Recall that the compact-open topology [3, p. 221] for $Y^{X}$ has as a subbase the totality of sets $(K, U) \cap Y^{X}$ where $K$ is a compact subset 
of $X$ and $U$ is an open subset of $Y$. Although the set $(K, U) \cap Y^{X}$ is always the same as $) K, U\left(\cap Y^{X}\right.$, the sets $(K, U)$ and $) K, U($ are in general distinct in $M(X, Y)$. It then seems very natural to make the following definition.

DeFinition 1.3. The compact-open topology for $M(X, Y)$ is the topology defined by taking the totality of $(K, U)$ and $) L, V($ as subbasic open sets, where $K$ and $L$ are any compact subsets of $X$ and $U$ and $V$ are any open sets in $Y$. The point-open topology for $M(X, Y)$ is the topology which has as a subbase the totality of the sets $(p, U)$ and $) p^{\prime}, U^{\prime}$, where $p$ and $p^{\prime}$ are points of $X$ and $U$ and $U^{\prime}$ are open in $Y$.

We recall that a family $\mathscr{F} \subset Y^{X}$ is said to be evenly continuous [3, p. 235] if and only if for each $x$ in $X$, each $y$ in $Y$, and each open neighborhood $V$ of $y$ there exist an open neighborhood $U$ of $x$ and an open neighborhood $W$ of $y$ such that if $f \in \mathscr{F}$ and $f(x) \in W$ then $f(U) \subset V$. The concept of even continuity has played an important role in [3, pp. 235-237] and [6]. We generalize this to any subset of $M(X, Y)$ :

DeFINITION 1.4. A family $\mathscr{F} \subset M(X, Y)$ is evenly continuous if and only if for each $x$ in $X$, each $y$ in $Y$, and each open neighborhood $V$ of $y$ there exist an open neighborhood $U$ of $x$ and an open neighborhood $W$ of $y$ such that:

(i) if $F \in \mathscr{F}$ and $F(x) \cap W \neq \square$ then $U \subset F^{-1}(V)$, and

(ii) if, in addition to (i), $F(x) \subset V$ then $F(U) \subset V$.

Using the notations we have introduced, the conditions (i) and (ii) above may be expressed equivalently as $\left(\mathrm{i}^{\prime}\right)$ and (ii'), respectively:

(i' ) $\mathscr{F} \cap) x, W(\subset) U, V($, and

(ii') $\mathscr{F} \cap) x, W(\cap(x, V) \subset(U, V)$.

It follows that if $\mathscr{F} \subset M(X, Y)$ consists entirely of single-valued functions, then $\mathscr{F}$ satisfies Definition (1.4) if and only if $\mathscr{F}$ is evenly continuous in the sense of Kelley [3, p. 235].

2. Properties of evenly continuous families of multifunctions.

Proposition 2.1. Every finite set of continuous multifunctions on $X$ to $Y$ is evenly continuous.

Proof. If $x$ is any point in $X$ and $\mathscr{F}=\left\{F_{1}, \cdots, F_{k}\right\}$ is a finite collection of continuous multifunctions on $X$ to $Y$, and if $y$ is any element of $Y$, let $V$ be any open neighborhood of $y$. Let $U_{1}=\bigcap_{i=1}^{n} F_{k_{i}}^{-1}(V)$, 
where $\left\{F_{k_{i}} \mid i=1, \cdots, n\right\}$ is the set of all $F$ in $\mathscr{F}$ such that $F(x) \cap V \neq \square$. (If $\left\{F_{k_{i}} \mid i=1, \cdots, n\right\}$ is empty, let $U_{1}=X$.) Let

$$
U_{2}=X-\bigcup_{j=n+1}^{n+m} F_{k_{j}}^{-1}(Y-V)
$$

where $\left\{F_{k_{j}} \mid j=n+1, \cdots, n+m\right\}$ is the set of all $F$ in $\mathscr{F}$ such that $x \notin F^{-1}(Y-V)$. (If $\left\{F_{k_{j}} \mid j=n+1, \cdots, n+m\right\}$ is empty, let $U_{2}=X$.) Since $\mathscr{F}$ contains only continuous multifunctions and since the union of a finite number of closed sets is closed and the intersection of a finite number of open sets is open, $U_{1}$ and $U_{2}$ are both open sets in $X$ containing $x$. Let $U=U_{1} \cap U_{2}$ and let $W=V$. Then $U$ and $W$ are open sets containing $x$ and $y$ respectively and, $F \in \mathscr{F} \cap) x, W(\Rightarrow F \in \mathscr{F}$ and $F(x) \cap W \neq \square$

$$
\begin{aligned}
& \Rightarrow F \in \mathscr{F} \text { and } F(x) \cap V \neq \square \\
& \Rightarrow F=F_{k_{i}}, \text { for some } i=1, \cdots, n \\
& \Rightarrow F^{-1}(V) \supset \bigcap_{i=1}^{n} F_{k_{i}}^{-1}(V)=U_{1} \supset U \\
& \Rightarrow F \in) U, V(.
\end{aligned}
$$

And, $F \in \mathscr{F} \cap) x, W(\cap(x, V) \Rightarrow F \in \mathscr{F} \cap(x, V)$

$$
\begin{aligned}
& \Rightarrow F \in \mathscr{F} \text { and } F(x) \subset V \\
& \Rightarrow F \in \mathscr{F} \text { and } x \notin F^{-1}(Y-V) \\
& \Rightarrow F=F_{k_{j}} \text {, for some } j=n+1, \cdots, n+m .
\end{aligned}
$$

Now if $z \in X-\bigcup_{j=n+1}^{n+m} F_{k_{j}}^{-1}(Y-V)$ then $z \in X-F^{-1}(Y-V)$ and hence $F(z) \cap(Y-V)=\square$. Thus, $F(z) \subset V$ and hence $F \in\left(U_{2}, V\right)$. So $F \in(U, V)$, since $U \subset U_{2}$. This shows that $\left.\mathscr{F} \cap\right) x, W(\subset) U, V($ and $\mathscr{F} \cap) x, W(\cap(x, V) \subset(U, V)$. Thus, $\mathscr{F}$ is evenly continuous.

Proposition 2.2. If $X$ has the discrete topology then $M(X, Y)$ is evenly continuous.

Proof. Let $x$ be any point of $X, y$ be any point in $Y$ and $V$ be any open neighborhood of $y$. Let $U=\{x\}$ and $W=V$ be open neighborhoods of $x$ and $y$ respectively. Then

$$
F \in M(X, Y) \cap) x, W(\Rightarrow F \in) x, V(\Rightarrow F \in) U, V(\text {. }
$$

And

$$
F \in M(X, Y) \cap) x, W(\cap(x, V) \Rightarrow F \in(x, V) \Rightarrow F \in(U, V) .
$$

Proposition 2.3. If $\mathscr{F} \subset M(X, Y)$ is evenly continuous and $F$ is any member of $\mathscr{F}$ then $F$ is a continuons multifunction on $X$ to $Y$.

Proof. Let $V$ be any open set in $Y$ and let $F$ be any member of $\mathscr{F}$ where $\mathscr{F}$ is an evenly continuous subset of $M(X, Y)$. If $F^{-1}(V)=\square$, then $F^{-1}(V)$ is open in $X$. If $F^{-1}(V) \neq \square$, then for all $x$ in $F^{-1}(V), F(x) \cap V \neq \square$, and for any $y_{x}$ in $F(x) \cap V$, by the even 
continuity of $\mathscr{F}$ there exist open neighborhoods $U_{x}$ and $W_{x}$ of $x$ and $y_{x}$ respectively such that $\left.F \in\right) U_{x}, V($ since $F \in \mathscr{F} \cap) x, W_{x}$ (. To see that $F \in) x, W_{x}\left(\right.$, note that $y_{x} \in F(x) \cap W_{x}$ and hence $F(x) \cap W_{x} \neq \square$. Thus, $U_{x} \subset F^{-1}(V)$ and hence $F^{-1}(V)$ is open. Now let $C=Y-V$. If $F^{-1}(C)=X$ then $F^{-1}(C)$ is closed in $X$. If $F^{-1}(C) \neq X$, then for all $x$ in $X-F^{-1}(C), F(x) \cap C=\square$. Thus, if $x$ is in $X-F^{-1}(C)$, then $F(x) \subset Y-C=V$ which is open in $Y$. By the even continuity of $\mathscr{F}$, for any $y_{x}$ in $F(x)$ there exist neighborhoods $U_{x}$ and $W_{x}$ of $x$ and $y_{x}$ respectively such that $F \in\left(U_{x}, V\right)$ since $\left.F \in \mathscr{F} \cap\right) x, W_{x}(\cap(x, V)$.

$$
\begin{aligned}
F \in\left(U_{x}, V\right) & \Rightarrow F\left(U_{x}\right) \subset V \Rightarrow F\left(U_{x}\right) \cap C=\square \Rightarrow U_{x} \cap F^{-1}(C) \\
=\square & \Rightarrow U_{x} \subset X-F^{-1}(C) .
\end{aligned}
$$

Thus $X-F^{-}(C)$ is open and $F^{-1}(C)$ is closed. This completes the proof.

Propositions (2.1) and (2.3) together say that a finite subset $\mathscr{F}$ of $M(X, Y)$ is evenly continuous if and only if $\mathscr{F}$ consists entirely of continuous multifunctions.

In the sequel, we denote by $M(X, Y ; c)$ the space $M(X, Y)$ equipped with the compact-open topology [see Definition (1.3)], and by $M(X, Y ; p)$ the space $M(X, Y)$ equipped with the point-open topology.

3. Ascoli's theorem for the space $M(X, Y ; c)$ of multifunctions.

From Proposition (2.2), if $X$ is a discrete space then $M(X, Y)$ is evenly continuous; and here the point-open topology and the compactopen topology coincide on $M(X, Y)$. The following lemma generalizes this occurrence.

Lemma 3.1. Let $\mathscr{F}$ be an evenly continuous subset of $M(X, Y)$. Then F has the same closure with respect to both the compact-open topology and the point-open topology; and these topologies induce the same topology in $\mathscr{F}$.

Proof. Let $\mathscr{G}$ be any subset of $\mathscr{F}$. Let $\mathscr{G}^{* c}$ and $\mathscr{G}^{* p}$ be the closures of $\mathscr{C}$ in $M(X, Y ; c)$ and $M(X, Y ; p)$ respectively. Since the point-open topology in $M(X, Y)$ is always contained in (=coarser than) the compact-open topology for $M(X, Y), \mathscr{G}^{* c} \subset \mathscr{G}^{* p}$. To show that $\mathscr{G}^{* c}=\mathscr{G}^{* p}$, it remains to show that $\mathscr{G}^{* p} \subset \mathscr{G}^{* c}$. To this end, let $F_{0}$ be any multifunction in $\mathscr{G}^{* p}$ and let $K_{1}, K_{2}, \cdots, K_{n}, L_{n+1}, \cdots, L_{n+m}$ be compact subsets of $X$ and $U_{1}, \cdots, U_{n} ; V_{n+1}, \cdots, V_{n+m}$ be open sets in $Y$ such that for all $i=1, \cdots, n, F_{0}\left(K_{i}\right) \subset U_{i}$ and for all 
$j=n+1, \cdots, n+m, L_{j} \subset F_{0}^{-1}\left(V_{j}\right)$.

Thus,

$$
\left.F_{0} \in\left(K_{1}, U_{1}\right) \cap \cdots \cap\left(K_{n}, U_{n}\right) \cap\right) L_{n+1}, V_{n+1}(\cap \cdots \cap) L_{n+m}, V_{n+m}(\cdot
$$

If $1 \leqq i \leqq n$, then since $\mathscr{G}$ is evenly continuous $(\mathscr{G} \subset \mathscr{F}$ and $\mathscr{F}$ is evenly continuous), for any $x \in K_{i}$ and for any $y_{x}$ in $F_{0}(x) \subset U_{i}$, there exist open neighborhoods $U_{x}$ and $W_{x}$ of $x$ and $y_{x}$ respectively such that $\mathscr{G} \cap) x, W_{x}\left(\cap\left(x, U_{i}\right) \subset\left(U_{x}, U_{i}\right)\right.$. Since $K_{i}$ is compact, $K_{i}$ is covered by a finite number of the open sets $U_{x}$. Let $\left\{U_{x_{(k, i)}} \mid k=\right.$ $1, \cdots, k(i)\}$ be such a finite open cover of $K_{i}$ for each $i=1, \cdots, n$; and for each $i=1, \cdots, n$ let $\left\{W_{x_{(k, i)}} \mid k=1, \cdots, k(i)\right\}$ be the set of corresponding open neighborhoods of points in $U_{i}$ existing by the even continuity of $\mathscr{G}$ such that for each $i=1, \cdots, n$ and for all $k=1, \cdots, k(i)$,

$$
\mathscr{G} \cap) x_{(k, i)}, W_{x_{(k, i)}}\left(\cap\left(x_{(k, i)}, U_{i}\right) \subset\left(U_{x_{(k, i)}}, U_{i}\right) .\right.
$$

If $n+1 \leqq j \leqq n+m$ and $x \in L_{j}$, then again by the even continuity of $\mathscr{G}$, for any $y_{x}$ in $F_{0}(x) \cap V_{j}$, there exist open neighborhoods $U_{x}$ and $W_{x}$ of $x$ and $y_{x}$ respectively such that $\left.\mathscr{G} \cap\right) x, W_{x}(\subset) U_{x}, V_{j}($. Since $L_{j}$ is compact, $L_{j}$ is covered by a finite number of the open sets $U_{x}$. Let $\left\{U_{x_{(q, j)}} \mid q=1, \cdots, q(j)\right\}$ be such a finite open covering of $L_{j}$ for each $j=n+1, \cdots, n+m$; and for each $j=n+1, \cdots, n+m$, let $\left\{W_{x_{(q, j)}} \mid q=1, \cdots, q(j)\right\}$ be the corresponding open neighborhoods of points in $F_{0}\left(x_{(q, j)}\right) \cap V_{j}$ existing by the even continuity of $\mathscr{G}$ such that $\mathscr{G} \cap) x_{(q, j)}, W_{x_{(q, j)}}(\subset) U_{x_{(q, j)}}, V_{j}($. Thus

and

$$
N_{1}=\overbrace{(k, i)=(1,1)}^{(k(i), n)}[) x_{(k, i)}, W_{x_{(k, i)}},\left(\cap\left(x_{(k, i)} U_{i}\right)\right]
$$

$$
N_{2}=\overbrace{(q, j)=(1, n+1)}^{(q(j), n+m)}) x_{(q, j)}, W_{x(q, j)}(
$$

are both basic open neighborhoods of $F_{0}$ in $M(X, Y ; p)$. And hence $N_{1} \cap N_{2}$ is an open neighborhood of $F_{0}$ in $M(X, Y ; p) . \quad F_{0} \in \mathscr{G}^{* p}$ implies that there exists a multifunction $F$ in $N_{1} \cap N_{2} \cap \mathscr{G}$. Since $\mathscr{G}$ is evenly continuous, $F \in \mathscr{G} \cap N_{1}$ implies that for each $i=1, \cdots, n$ and for each $k=1, \cdots, k(i), F \in\left(U_{x_{(k, i)}}, U_{i}\right)$; and $F \in \mathscr{G} \cap N_{2}$ implies that for each $j=n+1, \cdots, n+m$ and for each $q=1, \cdots, q(j)$, $F \in) U_{x_{(q, j)}}, V_{j}\left(\right.$. Since for each $i=1, \cdots, n,\left\{U_{x_{(k, i)}} \mid k=1, \cdots, k(i)\right\}$ is a cover of $K_{i}$, and for each $j=n+1, \cdots, n+m,\left\{U_{x_{(q, j)}} \mid q=\right.$ $1, \cdots, q(j)\}$ is a cover of $L_{j}$, we have $F \in\left(K_{i}, U_{i}\right)$ for each $i=1, \cdots, n$ and $F \in) L_{j}, V_{j}$ (for each $j=n+1, \cdots, n+m$. Thus,

$$
\left.F \in\left(K_{1}, U_{1}\right) \cap \cdots \cap\left(K_{n}, U_{n}\right) \cap\right) L_{n+1}, V_{n+1}(\cap \cdots \cap) L_{n+m}, V_{n+m}(.
$$

Since $\left.\left(K_{1}, U_{1}\right) \cap \cdots \cap\left(K_{n}, U_{n}\right) \cap\right) L_{n+1}, V_{n+1}(\cap \cdots \cap) L_{n+m}, V_{n+m}($ is an 
arbitrary basic open neighborhood of $F_{0}$ in $M(X, Y ; c)$ and this neighborhood always contains a member $F$ of $\mathscr{G}, F_{0}$ belongs to $\mathscr{G}^{* c}$. Since $F_{0}$ was an arbitrary element of $\mathscr{G}^{* p}$, we have shown $\mathscr{G}^{* p} \subset \mathscr{G}^{* c}$, and hence $\mathscr{G}^{* c}=\mathscr{G}^{* p}$. Since $\mathscr{G}$ was an arbitrary subset of $\mathscr{F}$, in particular $\mathscr{F}^{* c}=\mathscr{F}^{* p}$ and the subtopology for $\mathscr{F}$ induced by the compact-open topology for $M(X, Y)$ coincides with the subtopology for $\mathscr{F}$ induced by the point-open topology for $M(X, Y)$. This completes the proof of the lemma.

In [4], the cartesian $M$-product $P_{M}\left\{X_{\lambda} \mid \lambda \in \Lambda\right\}$ of the family $\left\{X_{\lambda} \mid \lambda \in \Lambda\right\}$ of spaces is defined to be the set of all multifunctions $F: \Lambda \rightarrow \cup\left\{X_{\lambda} \mid \lambda \in \Lambda\right\}$ such that $F(\lambda) \subset X_{\lambda}$ for each $\lambda \in \Lambda$; and the $M$-product topology for $P_{M}\left\{X_{\lambda} \mid \lambda \in \Lambda\right\}$ is the topology having as a subbase the totality of subsets $\left(\lambda, U_{\lambda}\right)$ and $) \mu, V_{\mu}$, where $U_{\lambda}$ and $V_{\mu}$ are any arbitrary open sets in $X_{\lambda}$ and $X_{\mu}$ respectively, and $\lambda$ and $\mu$ are any members of $\Lambda$. It is proved in $\left[4\right.$, p. 400] that if each $X_{\lambda}$ is compact then $P_{M}\left\{X_{\lambda} \mid \lambda \in \Lambda\right\}$ with the $M$-product topology is compact.

We now prove the main theorem.

THEOREM 3.2. Let $X$ and $Y$ be arbitrary topological spaces and let $M(X, Y ; c)$ be the space of all multifunctions on $X$ to $Y$ with the compact-open topology. Then a closed set $\mathscr{F}$ in $M(X, Y ; c)$ is compact if, at each point $x$ in $X, \mathscr{F}(x)=\cup\{F(x) \mid F \in \mathscr{F}\}$ has a compact closure in $Y$, and $\mathscr{F}$ is evenly continuous.

Proof. Suppose that $\mathscr{F}$ is a closed evenly continuous subset of $M(X, Y ; c)$ such that for each $x$ in $X, \mathscr{F}(x)$ has a compact closure in $Y$. Let $\mathscr{F}(x)^{*}$ denote the closure of $\mathscr{F}(x)$ for all $x$ in $X$, and let $Y_{x}=Y$ for all $x$ in $X$. Since $\mathscr{F}(x)^{*} \subset Y=Y_{x}$ for all $x$ in $X, P_{M}\left\{\mathscr{F}(x)^{*} \mid x \in X\right\} \subset P_{M}\left\{Y_{x} \mid x \in X\right\}=M(X, Y ; p)$. Since $\mathscr{F}(x)^{*}$ is compact for each $x$ in $X$, by Tychonoff's theorem for the space of multifunctions [4], $P_{M}\left\{\mathscr{F}(x)^{*} \mid x \in X\right\}$ is compact with the $M$-product topology. The $M$-product topology for $P_{M}\left\{\mathscr{F}(x)^{*} \mid x \in X\right\}$ coincides with the $M$-product topology for $P_{M}\left\{Y_{x} \mid x \in X\right\}$ relativized on the subset $P_{M}\left\{\mathscr{F}(x)^{*} \mid x \in X\right\}$, and the $M$-product space $P_{M}\left\{Y_{x} \mid x \in X\right\}$ is the same as the space $M(X, Y ; p)$. Consequently, the $M$-product space $P_{M}\left\{\mathscr{F}(x)^{*} \mid x \in X\right\}$ is a compact subspace of $M(X, Y ; p)$. Since for all $F \in \mathscr{F}, F(x) \subset \mathscr{F}(x)^{*}$ for every $x \in X$, we have

$$
\mathscr{F} \subset P_{M}\left\{\mathscr{F}(x)^{*} \mid x \in X\right\} \subset M(X, Y) \text {. }
$$

It then follows from Lemma (3.1) that $\mathscr{F}$ is closed in $M(X, Y ; p)$, and consequently it is closed in the compact space $P_{M}\left\{\mathscr{F}(x)^{*} \mid x \in X\right\}$. Thus, $\mathscr{F}$ is compact in $P_{M}\left\{\mathscr{F}(x)^{*} \mid x \in X\right\}$ and hence it is compact 
in $M(X, Y ; p)$. By Lemma (3.1), the compact-open subtopology and the point-open subtopology for $\mathscr{F}$ coincide. Therefore, $\mathscr{F}$ is compact in the space $M(X, Y ; c)$. This completes the proof of the theorem.

4. A remark. In the proof of Ascoli's theorem for multifunctions we make use of the Tychonoff theorem for multifunctions. It is interesting to observe that the Tychonoff theorem for multifunctions can be deduced directly from the Ascoli theorem for multifunctions, and hence these two theorems are, in a sense, equivalent. We now indicate a proof of this fact:

Let $\left\{Y_{x} \mid x \in X\right\}$ be a family of compact topological spaces, and let $Y^{x}=Y_{x} \times\{x\}$ for each $x \in X$. Then, $\left\{Y^{x} \mid x \in X\right\}$ is a family of disjoint spaces such that each $Y^{x}$ is homeomorphic with $Y_{x}$. It follows that the two $M$-product spaces $P_{M}\left\{Y_{x} \mid x \in X\right\}$ and $P_{M}\left\{Y^{x} \mid x \in X\right\}$ are homeomorphic. Now, let $X$ have the discrete topology and let $Y=\cup\left\{Y^{x} \mid x \in X\right\}$ be the free union of the spaces $Y^{x}, x \in X$. That is, a set $U \subset Y$ is open in $Y$ if and only if $U \cap Y^{x}$ is open in $Y^{x}$ for each $x$ in $X$. Let $\mathscr{F}=P_{M}\left\{Y^{x} \mid x \in X\right\} \subset M(X, Y)$. It can be shown that $\mathscr{F}$ with $M$ product topology is closed in $M(X, Y ; c)=M(X, Y ; p)$, because $X$ is discrete. Also, we have each $\mathscr{F}(x)=Y^{x}$ closed in $Y$, and hence each $\mathscr{F}(x)^{*}=Y^{x}$ which is compact. By Proposition (2.2), $M(X, Y)$ is evenly continuous and hence so is $\mathscr{F}$. Now Theorem (3.2) applies here to conclude that $\mathscr{F}$ is compact, and hence so is $P_{M}\left\{Y_{x} \mid x \in X\right\}$.

\section{BIBLIOGRAPHY}

1. G. Ascoli, Le curve limite di una varieta datà di curve, Mem. Accad. Lincei (3) 18 (1883), 521-586.

2. D. Gale, Compact sets of functions and function rings, Proc. Amer. Math. Soc. 1 (1950), 303-308.

3. J. L. Kelley, General topology, Van Nostrand, Princeton, 1955.

4. Y.-F. Lin, Tychonoff's theorem for the space of multifunctions, Amer. Math. Monthly 74 (1967), 399-400.

5. S. B. Myers, Equicontinuous sets of mappings, Ann. of Math. (2) 47 (1946), 496-502.

6. J. D. Weston, A generalization of Ascoli's theorem, Mathematika 6 (1959), 19-24.

Received November 21, 1969

The University of South Florida

TAMPA, FLORIDA 



\section{PACIFIC JOURNAL OF MATHEMATICS}

\section{EDITORS}

H. SAMELSON

Stanford University

Stanford, California 94305

\section{Richard Pierce}

University of Washington

Seattle, Washington 98105
J. DugundJI

Department of Mathematics

University of Southern California

Los Angeles, California 90007

RICHARD ARENS

University of California

Los Angeles, California 90024

\section{ASSOCIATE EDITORS}

\section{E. F. BeCKenBACH}

B. H. NeUmanN
K. YosHida

\section{SUPPORTING INSTITUTIONS}

\author{
UNIVERSITY OF BRITISH COLUMBIA \\ CALIFORNIA INSTITUTE OF TECHNOLOGY \\ UNIVERSITY OF CALIFORNIA \\ MONTANA STATE UNIVERSITY \\ UNIVERSITY OF NEVADA \\ NEW MEXICO STATE UNIVERSITY \\ OREGON STATE UNIVERSITY \\ UNIVERSITY OF OREGON \\ OSAKA UNIVERSITY \\ UNIVERSITY OF SOUTHERN CALIFORNIA
}

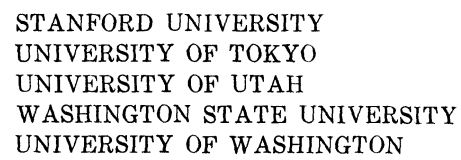

STANFORD UNIVERSITY UNIVERSITY OF TOKYO

UNIVERSITY OF UTAH

WASHINGTON STATE UNIVERSITY

UNIVERSITY OF WASHINGTON

The Supporting Institutions listed above contribute to the cost of publication of this Journal, but they are not owners or publishers and have no responsibility for its content or policies.

Mathematical papers intended for publication in the Pacific Journal of Mathematics should be in typed form or offset-reproduced, (not dittoed), double spaced with large margins. Underline Greek letters in red, German in green, and script in blue. The first paragraph or two must be capable of being used separately as a synopsis of the entire paper. The editorial "we" must not be used in the synopsis, and items of the bibliography should not be cited there unless absolutely necessary, in which case they must be identified by author and Journal, rather than by item number. Manuscripts, in duplicate if possible, may be sent to any one of the four editors. Please classify according to the scheme of Math. Rev. Index to Vol. 39. All other communications to the editors should be addressed to the managing editor, Richard Arens, University of California, Los Angeles, California, 90024.

50 reprints are provided free for each article; additional copies may be obtained at cost in multiples of 50 .

The Pacific Journal of Mathematics is published monthly. Effective with Volume 16 the price per volume (3 numbers) is $\$ 8.00$; single issues, $\$ 3.00$. Special price for current issues to individual faculty members of supporting institutions and to individual members of the American Mathematical Society: $\$ 4.00$ per volume; single issues $\$ 1.50$. Back numbers are available.

Subscriptions, orders for back numbers, and changes of address should be sent to Pacific Journal of Mathematics, 103 Highland Boulevard, Berkeley, California, 94708.

PUBLISHED BY PACIFIC JOURNAL OF MATHEMATICS, A NON-PROFIT CORPORATION

Printed at Kokusai Bunken Insatsusha (International Academic Printing Co., Ltd.), 7-17, Fujimi 2-chome, Chiyoda-ku, Tokyo, Japan. 


\section{Pacific Journal of Mathematics}

\section{Vol. 34, No. 3 \\ July, 1970}

Richard Hindman Bouldin, The peturbation of the singular spectrum

Hugh D. Brunk and Søren Glud Johansen, A generalized Radon-Nikodym derivative .

Henry Werner Davis, F. J. Murray and J. K. Weber, Families of $L_{p}$-spaces

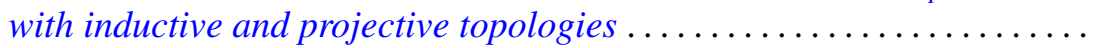

Esmond Ernest Devun, Special semigroups on the two-cell .

Murray Eisenberg and James Howard Hedlund, Expansive automorphisms

of Banach spaces ......................................

Frances F. Gulick, Actions of functions in Banach algebras.

Douglas Harris, Regular-closed spaces and proximities.

Norman Lloyd Johnson, Derivable semi-translation planes . .

Donald E. Knuth, Permutations, matrices, and generalized Young

tableaux..........................................

Herbert Frederick Kreimer, Jr., On the Galois theory of separable

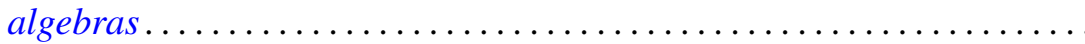

You-Feng Lin and David Alon Rose, Ascoli's theorem for spaces of

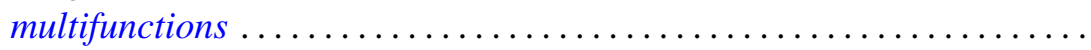

David London, Rearrangement inequalities involving convex functions . . . .

Louis Pigno, A multiplier theorem.

749

Helga Schirmer, Coincidences and fixed points of multifunctions into trees.

755

Richard A. Scoville, Some measure algebras on the integers .

Ralph Edwin Showalter, Local regularity of solutions of Sobolev-Galpern

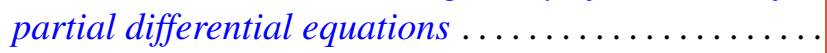

Allan John Sieradski, Twisted self-homotopy equivalences

John H. Smith, On S-units almost generated by S-units of subfields ...

803

Masamichi Takesaki, Algebraic equivalence of locally normal

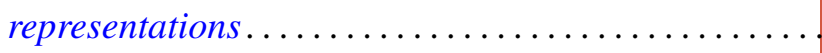

Joseph Earl Valentine, An analogue of Ptolemy's theorem and its converse in

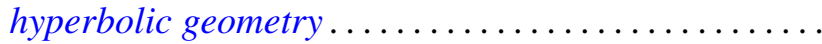

David Lawrence Winter, Solvability of certain p-solvable linear groups of finite order 